\title{
AC POWER SUPPLY FOR WOBBLER MAGNET OF THE MC-50 CYCLOTRON*
}

\author{
Y. S. Kim, J. S. Chai, Cyclotron Application Laboratory, KIRAMS, Seoul, Korea \\ K. H. Park\#, W. W. Lee, H. G. Lee, C. W. Chung, PAL, POSTECH, Pohang, Korea, \\ B. K. Kang, Department of Electrical Engineering, POSTECH, Pohang, Korea
}

\section{Abstract}

A wobbler magnet and an AC power supply for the MC-50 cyclotron $(\mathrm{k}=50)$ at Korea Institute of Radiological and Medical Sciences (KIRAMS) were designed and constructed to increase the area of beam irradiation. The power supply has two independently operating channels for the vertical and horizontal coils of the wobbler magnet. The frequency of the $\mathrm{AC}$ power supply for both coils is programmable from 1 to $20 \mathrm{~Hz}$ in a step of $1 \mathrm{~Hz}$, and the maximum output current is $12 \mathrm{~A}$ rms. With the wobbler magnet, the uniform irradiation area of the proton beam increased $\sim 4$ times larger than the one without the magnet, and various properties of the magnet and power supply system indicate that the system is well suited for many ion beam experiments.

\section{INTRODUCTION}

The MC-50 cyclotron at KIRAMS produces the ion beam for nuclear physics, chemistry, and applied researches in Korea. It has a small beam diameter with Gaussian beam shape, whereas many users want a beam irradiation on a large target with same beam current. A wobbler magnet system has been applied in the heavy ion experiments [1-2]. It is a beam delivery system using two dipole magnets to produce a large irradiation field. The simple principle of the wobbler magnet system is shown in Fig.1. The injected ion beam is scanned using the wobbler magnets so that experiments can be carried out with high efficiency [3].

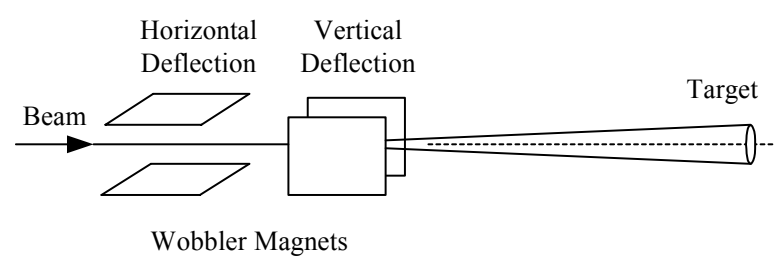

Figure 1: Working principle of the wobbler magnet system.

The fabricated wobbler magnet is a combined function magnet, which has a return yoke with two sets of coils: one coil set for the vertical deflection and the other for the horizontal deflection. Each coil set of magnet is driven separately using a linear magnet power supply. The power supply has no output filter for switching noise rejection. In this paper, the major parts and test results of the power supply system are described.

\footnotetext{
* Supported by the Ministry of Science and Technology of Korea

\#pkh@postech.ac.kr
}

\section{SYSTEM CONFIGURATION}

The system consists of a PLC for general control, a DAC/sine wave generator, an error and a power amplifiers, and a touch screen for displaying the statuses and accepting commands, as shown in Fig. 2.

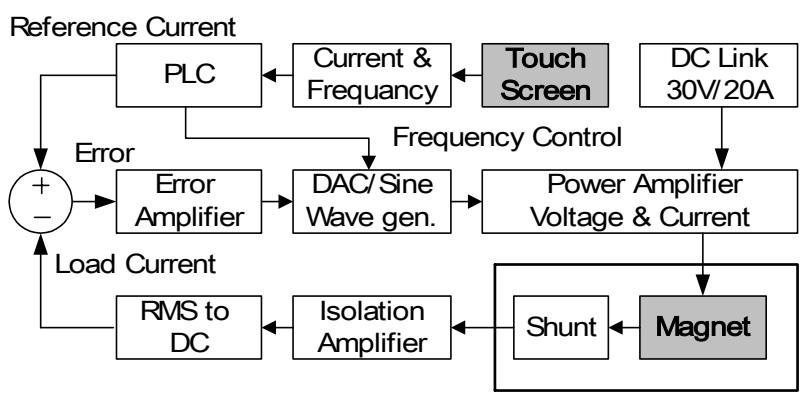

Figure 2: System block diagram.

The reference input of an error amplifier is set by the PLC which accepts the desired current value from the touch screen or a computer through the RS232C. The CPU C8051F060 from the Silicon Laboratories and the DAC1210 from National Semiconductor Co. on the DAC/sine wave generator outputs a sinusoidal waveform of a desired frequency. The two magnet power supplies for vertical and horizontal coils need to be controlled separately for the following parameters: the amplitude of $0-13 \mathrm{~A} \mathrm{rms}$, frequency of $1-20 \mathrm{~Hz}$, and phase of 0 $359^{\circ}$. All these required controls are made on the $\mathrm{DAC} /$ sine wave generator. The power amplifier amplifies the output of DAC/sine wave generator and drives the magnet. The output waveforms of two magnet power supplies are synchronized to each other, and various shapes of irradiation patterns are obtained by adjusting the control parameters. The power amplifier consists of a voltage amplifier, which was implemented using a PA08A voltage amplifier from APEX Microtechnology Co., and a current amplifier, which was implemented using two types of MJ15011 and MJ15012 complementary transistors from $\mathrm{ON}$ semiconductor Co. The AC load current was measured using a shunt resistor of $0.2 \Omega$ and the voltage across the shunt resistor was converted into a DC voltage using an AD536 RMS-toDC converter from Analog Devices Co. This DC voltage was compared with the reference current signal from PLC at the error amplifier, which produces a reference voltage to the DAC1210. The output current of the power amplifier was adjusted until the error signal became zero. 
The output frequency of the magnet power supply needs to be adjusted in the range of $1-20 \mathrm{~Hz}$ at a step of $1 \mathrm{~Hz}$. When the frequency changes, the input voltage to the power amplifier has to be adjusted to keep the magnet current constant. The error amplifier can do the required voltage adjustment. However, to increase the system reliability, the maximum input voltage to the power amplifier was increased with an increase of frequency using the frequency compensation circuits shown in Fig. 3. The simulated and measured frequency responses of the circuit are shown in Fig.4.

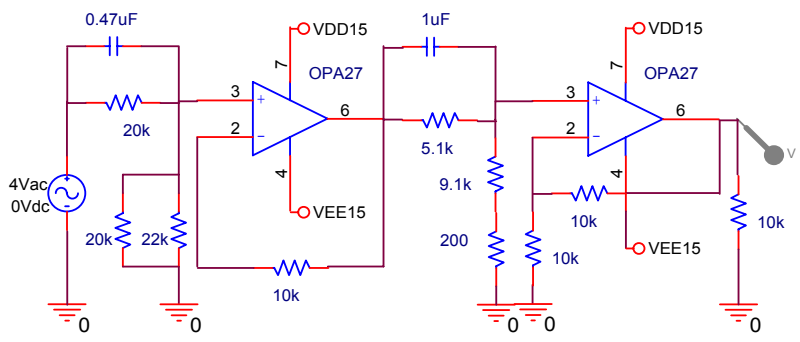

Figure 3: Frequency compensation circuits for the control signal.

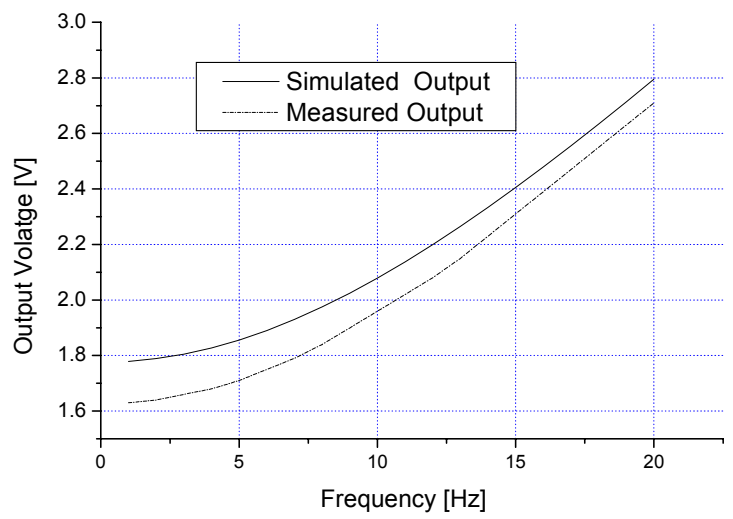

Figure 4: Simulated and measured frequency responses of the frequency compensation circuits.

The algorithm for generating a sinusoidal voltage waveform, which was provided by the Silicon Laboratories [4], has been modified to meet the design goals. A lookup table for two periods of sine wave was stored in the CPU C8051F060. On this table, one period is divided into 256 components using the 8-bit table indices, as shown in Fig. 5. When the waveform for vertical coil starts at a given offset from the reference index, where the waveform for horizontal coil starts, the vertical waveform can have a phase offset in the range of $0-355^{\circ}$ from the one for the horizontal waveform. The vertical and horizontal waveform generators use the same timing clock and the phase synchronization between these two waveforms is achieved. The frequency of waveform changes when the time interval for increment of table index changes. The resolution of phase difference between two waveforms is $360^{\circ} / 256=$ $1.4^{\circ}$.

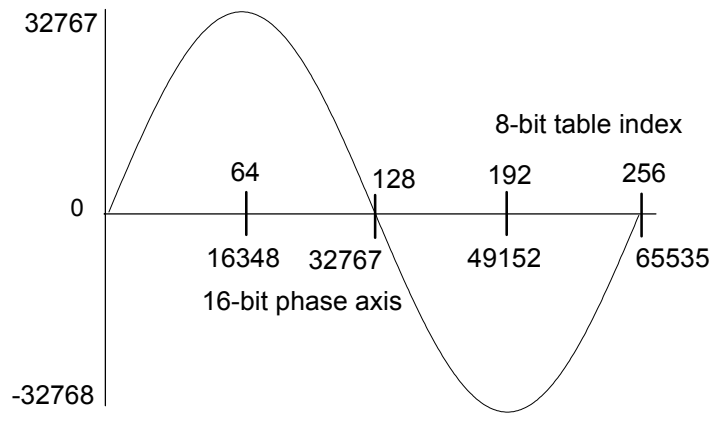

Figure 5: Lookup table for one period of sine wave.

\section{MEASUREMENT RESULTS}

A pickup-coil was fixed in the pole gap of the magnet to measure the magnetic field of the wobbler magnet. This coil is a 46-turn coil with a width of $1.15 \mathrm{~cm}$ and a length longer than the length of magnet. The voltage induced at the coil was integrated using an analog integrator, which is implemented using an OPA27 operation amplifier. The gain of the integrator was 537.6. The offset voltage of the amplifier was nullified before the start of field measurement. The bottom trace of Fig. 6 shows the integrated voltage output of the pickup coil when the horizontal coil of magnet is excited with a sine wave shown at the top trace of Fig. 6, which has a frequency of $1 \mathrm{~Hz}$ and amplitude of $7 \mathrm{~A}$. The measured peak of field integral along the beam direction was 47.9 G-m, which agrees well with the result of the Hall probe measurement; the measured peak magnetic field using a Hall probe was $310 \mathrm{G}$ and the effective length of the magnet was $0.15 \mathrm{~m}$.

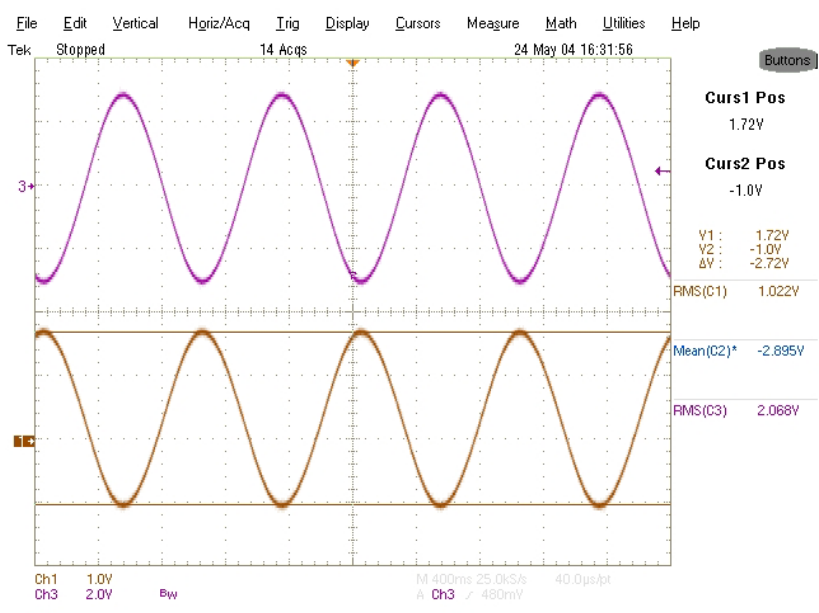

Figure 6: The current waveform (top trace) and the integrated voltage output of the pickup coil (bottom trace).

The inductance and resistance of the magnet were 1.5 $\mathrm{mH}$ and $0.025 \Omega$. A shunt resistor of $0.2 \Omega$ was 
connected in series with the magnet coil to sense the magnet current and the voltage across the resistor was feed to the feedback loop. When the magnet was excited with a sinusoidal current with a frequency of $1 \mathrm{~Hz}$ and amplitude of $13 \mathrm{~A}$, the voltage across the magnet and the negative DC voltage for the output stage of power amplifier are shown in Fig. 7. From Fig.7, one observes that the output current (channel 3) is a good sinusoidal waveform and the negative DC voltage for the output stage of power amplifier drops $\sim 5 \mathrm{~V}$.

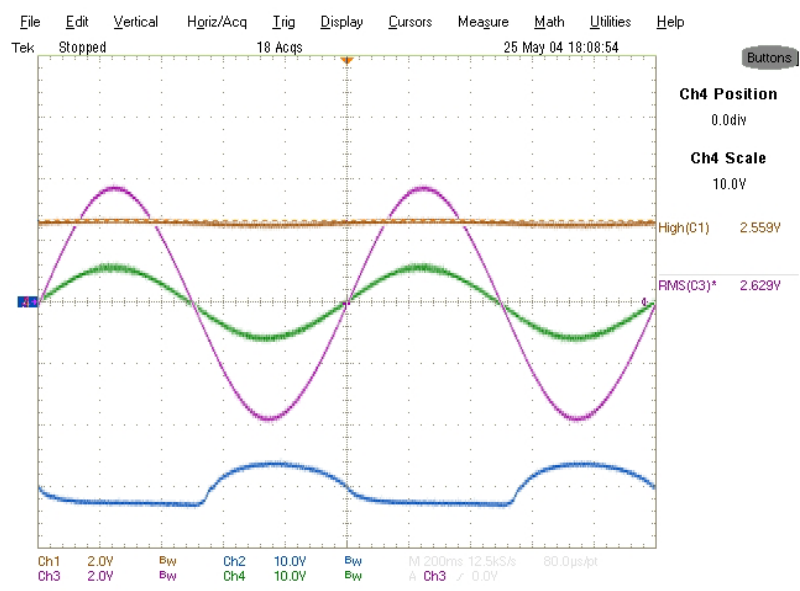

Figure 7: Measured various waveforms when frequency is $1 \mathrm{~Hz}$, load current is 13 Arms: current set command (ch1), voltage drop at -DC link (ch2), load current waveform (ch3) and voltage drop at load (ch4), respectively.

To measure the background current noise level, the output ripple voltages at the vertical and horizontal shunt resistors were measured while setting the reference input current to zero, and the results are shown in Fig. 8.

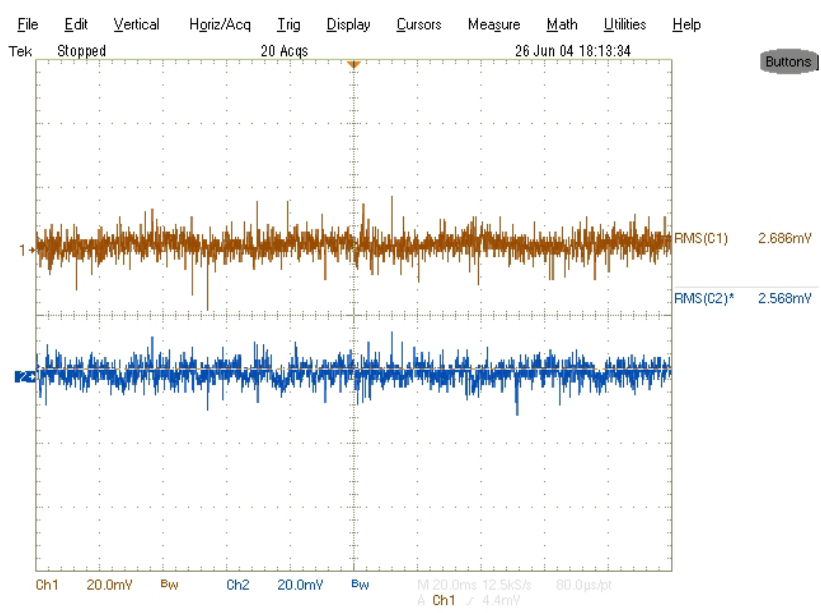

Figure 8: The measured noise voltages at the shunt resistors.

The measured noise voltages were $\sim 2.5 \mathrm{mV}$, which corresponds to $12.5 \mathrm{mArms}$ because the shunt resistance is $0.2 \Omega$. The noise-to-signal ratio at the output current of 13 A rms is $-60 \mathrm{~dB}$ approximately.

The irradiated beam profile with the wobbler magnet was measured using a radiochromic densitometer at the target and the result is shown in Fig. 9. The irradiated area was $\sim 4$ times larger than the one obtained without the wobbler magnet.

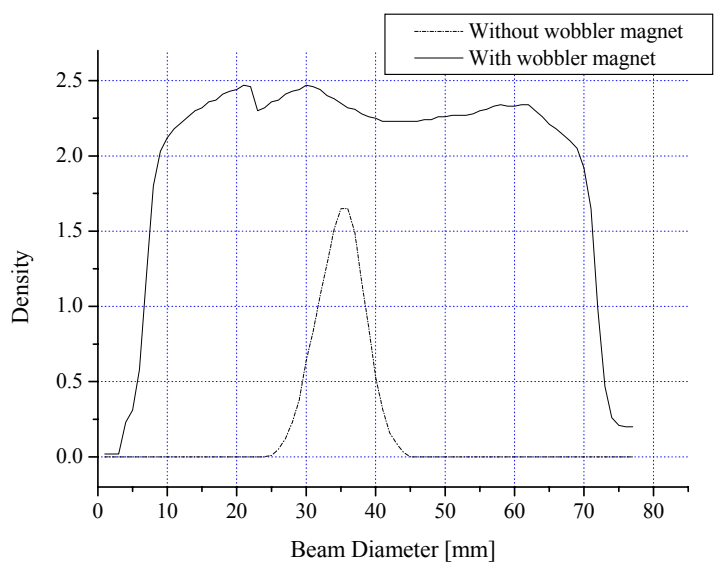

Figure 9: Measured $\mathrm{x}$ axis irradiated beam profiles with and without the wobbler magnet, when $\mathrm{x}$ was $1 \mathrm{~Hz}$ and $\mathrm{Y}$ $10 \mathrm{~Hz}$ wobbling.

\section{CONCLUSIONS}

For the MC-50 cyclotron, two AC power supplies for the horizontal and vertical coils of wobbler magnet were built and tested. The amplitude, frequency, and phase of these two power supplies were controlled separately, while the phase was synchronized. With the synchronized operation in phase, various shapes of irradiation patterns are obtained by adjusting the control parameters. The irradiated area with the wobbler magnet was $\sim 4$ times larger than the one without the wobbler magnet, which is sufficient for most experiments with MC-50 cyclotron at KIRAMS.

\section{REFERENCES}

[1] Tatsuaki Kanai, et al., "An irradiation facility and a beam simulation program for proton radiation therapy", Nuclear Instruments and Methods in Physics Research A302, 1991, p. 158-164.

[2] W. T. Chu, et al., "Wobbler Facility for Biomedical Experiments at the BEVALAC", IEEE Tran. on Nuclear Science, Vol.NS-32. No. 5, October 1985, p. 3321-3323

[3] Tatsuaki Kanai, et al., "Biophysical Characteristics of HIMAC Clinical Irradiation System for HeavyIon Radiation Therapy", Int. J. Radiation Oncology Biol. Phys., Vol. 44, No.1, 1999, p. 201-210 